\title{
Spectroscopic Phenomenological Estimation of the Functional State of the Human Organism in Rate and Pathology
}

\author{
Michail Yurevich Dolomatov ${ }^{2}$, Nikolay Vasilevich Kalashchenko ${ }^{1}$, Sergei Vladislavovich Dezortsev ${ }^{2}$, \\ Timur Ramilevich Araslanov ${ }^{1,2}$
}

${ }^{1}$ Bashkiria State Medical University, Ufa, Russia; ${ }^{2}$ Department of Physics, Ufa State Academy of Economics and Service, Ufa, Russia. Email: realitim@yandex.ru

Received October $23^{\text {rd }}, 2010$; December $10^{\text {th }}, 2010$; February $12^{\text {th }}, 2011$.

\begin{abstract}
In laboratory diagnostics uses a large amount of blood parameters to determine the functional state of the human organism. To our opinion, the evaluation may be given by the phenomenological electron spectroscopy analysis of biological liquids, which regards blood and its components as one and indivisible light-absorbing system without separating into individual compounds. Blood, plasma and serum solutions from donors and groups of patients (with pyoinflammatory diseases, therapeutic reanimation state, with renal insufficiency and cirrhosis) were studied. The difference of phenomenological characteristics of the spectra in rate and pathology was found. Results were confirmed by statistical data processing.
\end{abstract}

Keywords: Phenomenological, Absorption, Spectroscopy, Blood, Spectrum

\section{Introduction}

Methods of spectroscopy are widely used for the study of biological fluids. The authors suggested a new approach to research functional state of human organism based on electron spectrum of blood. This is an unusual aspect of electron spectroscopy has been named the electron phenomenological spectroscopy [1]. Contrary to conventional methods phenomenological spectroscopy studies substance as one indivisible system, without separating spectrum of the substance into characteristic frequencies and wavelengths of individual functional groups of components of the system.

The phenomenological approach may be used for determination the average characteristic-indexes of electronic structure for the whole system were determined on the analogy with indexes of reaction capability in chemistry of "pure substances" [1]. The problem is solved on the basis of discovered connecting between integral strength of oscillators (the area under curve of absorption of radiation in visible and UV diapason of spectrum) with ionization potentials (IP) and affinity to electron (AE) [2]. In the course of statistical research of several hundreds of atoms spectrums and organic molecules the correlation of IP and AE and integral oscillator force was established.

Except IP and AE are used three parameters of identification: the probability of light-absorbance, the factor of intensity of light-absorbance and the factor of fine structure of spectrum [2].

The integral characteristic, determined in the case of biological objects, differs from the absorption factor of molecular solutions, because absorption effects of dispersion and fluorescence are taking place. That is why the integral characteristic determined may be regarded as effective absorption factor of biological system.

In presented work were determined blood and its components as a system of quasiparticles in excited state. This way, all of physicochemical properties of such kind of system are formed by its effective energy characteristics, connected with absorption spectrums in UV and VIS radiation.

\section{Experimental Procedure}

We have examined 100 donors and 180 ailing people with different diagnosis and disease severity level. Absorption spectroscopy analysis of blood, plasma and serum solutions on the authoring method was carried out. Distilled water was taken as a vehicle. To determine ab- 


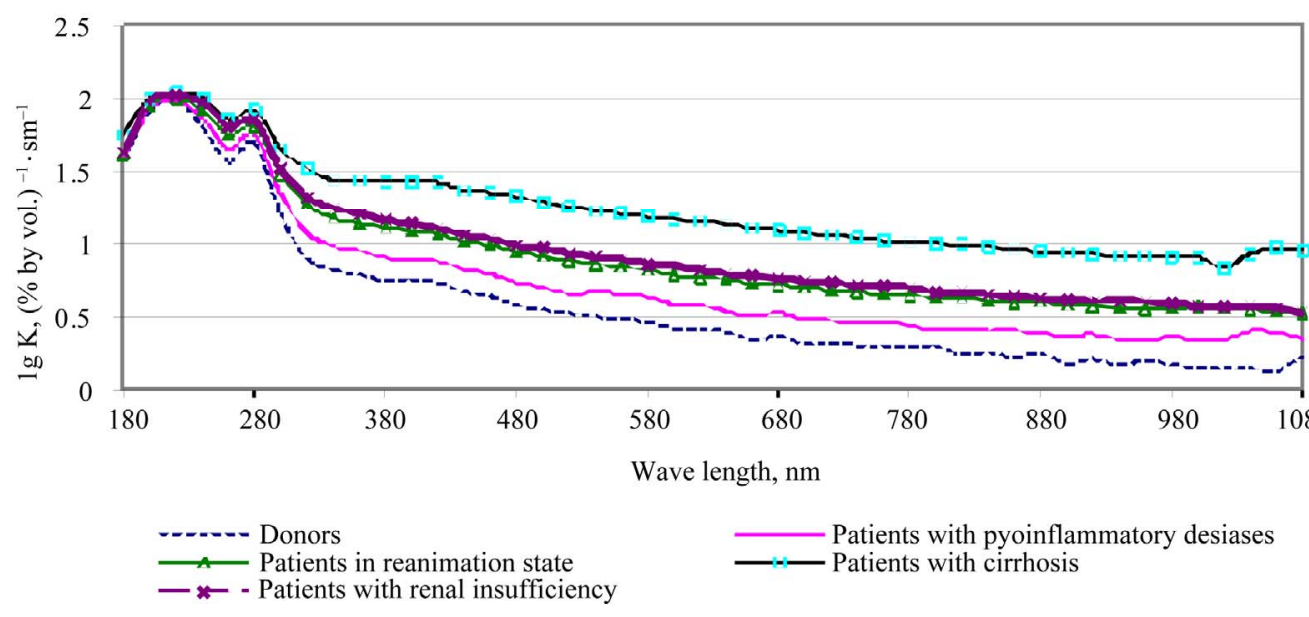

Figure 1. Four patient groups in comparison with donors (average spectrums of plasma subsystem).

Table 1. Phenomenological parameters of the absorption spectrums of plasma subsystem for donors and four groups of patients.

\begin{tabular}{|c|c|c|c|c|c|}
\hline & \multicolumn{5}{|c|}{ Plasma } \\
\hline & \multirow{2}{*}{ Donors } & \multicolumn{4}{|c|}{ Patients } \\
\hline & & Pyoinflammatory diseases & Reanimation state & Renal insufficiency & Cirrhosis \\
\hline \multicolumn{6}{|c|}{ Probability of light-absorbance, $-p \cdot 10^{3}, \mathrm{~nm}^{-1}$} \\
\hline Average value & 4.7 & 4.651 & 4.362 & 4.46 & 3.356 \\
\hline Confidence interval $\alpha=0.95$ & 0.16 & 0.26 & 0.14 & 0.14 & 0.384 \\
\hline Variation coefficient & 16.17 & 22.07 & 10.45 & 10.64 & 32.34 \\
\hline$\Delta, \mathrm{nm}^{-1}$ & -- & 0.045 & 0.334 & 0.24 & 1.34 \\
\hline \multicolumn{6}{|c|}{ Intensity of light-absorbance, $\times 10^{1},(\% \text { by vol. })^{-1} \cdot \mathrm{sm}^{-1}$} \\
\hline Average value & 64.63 & 92.13 & 101.61 & 112.24 & 128.8 \\
\hline Confidence interval $\alpha=0.95$ & 5.35 & 6.88 & 11.63 & 8.95 & 14.5 \\
\hline Variation coefficient & 39.66 & 29.52 & 37.38 & 26.67 & 22.96 \\
\hline$\Delta,(\% \text { by vol. })^{-1} \cdot \mathrm{sm}^{-1}$ & -- & -27.5 & -36.98 & -47.61 & -64.17 \\
\hline \multicolumn{6}{|c|}{ Factor of fine structure, $(\% \text { by vol. })^{-1} \cdot \mathrm{sm}^{-1}$} \\
\hline Average value & 37.46 & 30.43 & 27.36 & 1593.09 & 16.12 \\
\hline Confidence interval $\alpha=0.95$ & 2.1 & 1.87 & 2.59 & 115.92 & 2.98 \\
\hline Variation coefficient & 26.87 & 24.25 & 30.88 & 24.34 & 37.63 \\
\hline$\Delta,(\% \text { by vol. })^{-1} \cdot \mathrm{sm}^{-1}$ & -- & -7.03 & -10.1 & 1555.63 & -21.34 \\
\hline \multicolumn{6}{|c|}{ Integral oscillator strength (IOS), $\theta, 10^{-7} \cdot(\% \text { by vol. })^{-1}$} \\
\hline Average value & 409.2 & 518.8 & 552.9 & 578.49 & 746.6 \\
\hline $\begin{array}{c}\text { Confidence interval } \\
\alpha=0.95\end{array}$ & 21.2 & 28.5 & 39.2 & 29.41 & 61.0 \\
\hline Variation coefficient & 24.89 & 21.68 & 23.16 & 17.01 & 16.67 \\
\hline$\Delta, 10^{-7} \cdot(\% \text { by vol. })^{-1}$ & -- & 109.6 & 143.7 & 167.2 & 337.4 \\
\hline \multicolumn{6}{|c|}{ Effective ionization potential, eV } \\
\hline Average value & 8.529 & 8.26 & 8.179 & 8.12 & 7.72 \\
\hline $\begin{array}{c}\text { Confidence interval } \\
\alpha=0,95\end{array}$ & 0.503 & 0.067 & 0.093 & 0.08 & 0.15 \\
\hline Variation coefficient & 2.95 & 3.24 & 3.72 & 3.14 & 3.84 \\
\hline$\Delta, \mathrm{eV}$ & -- & -0.269 & -0.35 & -0.41 & -0.811 \\
\hline \multicolumn{6}{|c|}{ Effective electron affinity, eV } \\
\hline Average value & 0.51 & 0.617 & 0.651 & 0.686 & 0.84 \\
\hline $\begin{array}{c}\text { Confidence interval } \\
\alpha \alpha=0.95\end{array}$ & 0.207 & 0.028 & 0.038 & 0.03 & 0.06 \\
\hline Variation coefficient & 20.39 & 27.69 & 29.07 & 15.46 & 19.63 \\
\hline$\Delta, \mathrm{eV}$ & -- & 0.107 & 0.141 & 0.176 & 0.33 \\
\hline
\end{tabular}


sorbtion spectrums in wave-length interval from 180 to $1080 \mathrm{~nm}$ with step $20 \mathrm{~nm}$ we made solutions of 2.5 percent by volume concentration $(1: 40)[3,4]$.

\section{Experimental Results}

Differences between average spectrums of blood components in groups of patients (with pyoinflammatory diseases, therapeutic reanimation state, with renal insufficiency and cirrhosis) and average spectrums of blood components of donors especially became apparent for plasma (Figure 1).

Parameters of identification, integral strength of oscillator and effective (average value of all light-absorbing components) ionization potential were estimated in interval from $240 \mathrm{~nm}$ to $800 \mathrm{~nm}$. Estimated integral parameters of plasma system of human blood in conditions of homeostasis (donors) and deflections from homeostasis (patients) are presented in Table 1.

Parameters of average effective electronic structure of plasma (effective potential of ionization and electron affinity) were calculated on the base of empiric factors, estimated for model compounds of amino acids [5]. Evident differences may be observed for the donors and in the cases of different groups of patients.

\section{Discussion}

Thus, correlations between functional state of human organism and deflection of phenomenological spectrum parameters of plasma from the homeostasis state have been determined. Most reliable results may be observed for absorption spectrums of plasma in interval from 240 $\mathrm{nm}$ to $800 \mathrm{~nm}$. It is offered to use aberrations from relative "average" standard, calculated on the basis of donors, for estimation of condition of ailing or healthy organism.

\section{Conclusions}

Phenomenological approach enables express-evaluation of the functional state of health of human with specific phenomenological spectroscopy parameters. Such as integral strength of oscillator, effective ionization potential, electron affinity parameters of identification [6].

\section{REFERENCES}

[1] M. Yu. Dolomatov, "Fragment of the Theory of Real Substance. From Hydrocarbonic Systems to Galaxies: Moscow: Chemistry," 2005, p. 208.

[2] M. Yu. Dolomatov, "Chemical Physics of Multicomponent Organic Systems," Institute of Petroleum Refining and Petrochemistry Problems, Technology Institute of Service, Ufa, 2000, p. 124

[3] N. V. Kalashchenco, M. Yu. Dolomatov, S. V. Dezortsev, E. A. Popova and R. R. Kurmankaeva, "Peculiarities of Integral Characteristics of Electronic Spectrums of Donor Blood in UV and VIS Areas of Electromagnetic Spectrum," The Bashkir Chemical Journal. Vol. 11, No. 2, 2004, pp. 47-50.

[4] M. Yu. Dolomatov, N. V. Kalashchenco, S. V. Dezortsev, A. A. Korjenevsky, E. A. Popova and R. R. Kurmankaeva, "Studies of Integral Characteristics of Electronic Spectrums of Blood in Purulent Deseases," The Bashkir Chemical Journal, Vol. 11, No. 4, 2004, pp. 35-37.

[5] M. Yu. Dolomatov, N. V. Kalashchenco, S. V. Dezortsev, R. A. Chisamutdinov, T. M. Oshnyakova and M. S. Pavlova, "Evaluation of Average Characteristics of Electronic Structure of Biological Systems with Phenomenological Methods," Bashkir Chemical Journal, Vol. 13, No. 5, 2006, pp. 42-46.

[6] N. V. Kalashchenko, M. Yu. Dolomatov and S. V. Dezortsev, "The Electronic Phenomenological Spectroscopy Blood of Human in Rate and Pathology. Theory and Practice Aspects," Inter, Moscow, 2010, p. 256. 